\title{
TRANSNASAL TOPICAL SPHENOPALATINE GANGLION BLOCK TO TREAT CHRONIC DRUG- RESISTANT MIGRAINE: A CASE REPORT
}

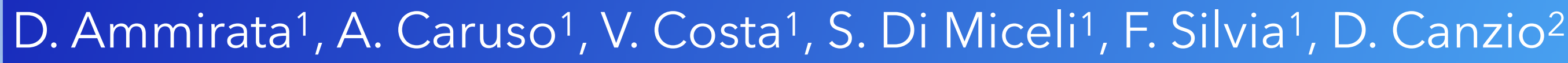

1Section of Anaesthesia- Analgesia- Emergency- Intensive Care. Palermo University Medical Center- Italy. A. Giarratano, Full Professor

2Department of Surgical- Oncological and Oral Science Di.Chir.On.S., Palermo- Via del Vespro 129. 90127, Italy.

\section{BACKGROUND}

The history of sphenopaltine ganglion nerve block dates to 1908 when Greenfield Sluder, M.D., first described the treatment of a variety of headache and facial pain syndromes by blocking this sympathetic ganglion with cocaine. SPG blocks are a proposed treatment option for chronic migraines and some severe non migraines and some severe non-migraine headaches. The SPG is a group of nerve cells located behind the bony structures of the nose. The nerve bundle is linked to the trigeminal nerve, the primary nerve involved in headache disorders. The rationale for using SPG blocks to treat headaches is that local anesthetics in low concentrations could block the sensory fibers and thereby reduce pain while maintaining autonomic function.

We report e seccessful case of the Tx360 ${ }^{\circledR}$ Nasal Applicator (Tian Medical) employment in a patient with several chronic migraine

\section{CLINICAL PRESENTATION}

A caucasian 38-year-old woman with a long history of chronic migraine with trigeminal pattern, BMI 27, no smoking history, without comorbidity, was referred to our pain clinic because of intractable pain. For analgesia amitriptyline, clonazepam, ergotamine, non-steroidal anti-inflammatory drugs had been tried but had produced minimal benefit. On physical examination, she had no sensory or motor deficit, and her cranial nerves were intact. She had considerable pain on light touch of the skin overlying the nose, the maxillae, and the forehead. We initiated a trial of SPGB by instillation using Tx360 ${ }^{\circledR}$ Nasal Applicator (Tian Medical). With patient in seated position, we inserted the catheter intranasally and, once in place, we instilled 7,5\% levobupivacaine, $3 \mathrm{ml}$ into each nostril. After 20 minutes she reported a diminution of her pain by at least $50 \%$ as indicated on a visual analog scale from 0 to 10 . We repeated the treatment twice a week for 3 weeks (total 6 treatments). On subsequent follow-up visits over a period of 3 months, she reported a consistently reduction of her pain by $50 \%$ to $60 \%$. Local skin tenderness over her nose decreased considerably and over her maxillae and eyes to some extent. The patient did not experience any disorientation or other symptoms suggesting systemic local anestetic toxicity.

\section{CONCLUSION}

This clinical observation suggest that repetive SPG blocks using levobupivacaine may be an effective treatment for chronic, resistant migraine. The $T \times 360^{\circledR}$ Nasal Applicator is the only device with which patient remain seated position, reducing discomfort during instillation

\section{REFERENCES}

Are repetitive pericranial nerve blocks effective in the management of chronic paroxysmal hemicrania?: A case report. Ertem DH. Medicine (Baltimore). 2019.; Intranasal self-administration of local anesthetic (ropivacaine) for sphenopalatine ganglion block, for treatment of second trigeminal branch neuralgia secondary to maxillary sinus curettage: $\boldsymbol{A}$ case report. Lima MH, et al. Rev Esp Anestesiol Reanim. 2019; Transnasal Sphenopalatine Ganglion Block for Postdural Puncture Headache in an Adolescent: A Case Report; Stalls C, et al. A A Pract. 2019. Sphenopalatine Ganglion Block and Radiofrequency Ablation: Technical Notes and Efficacy.Review article. Tolba R, et al. Ochsner J. 2019.

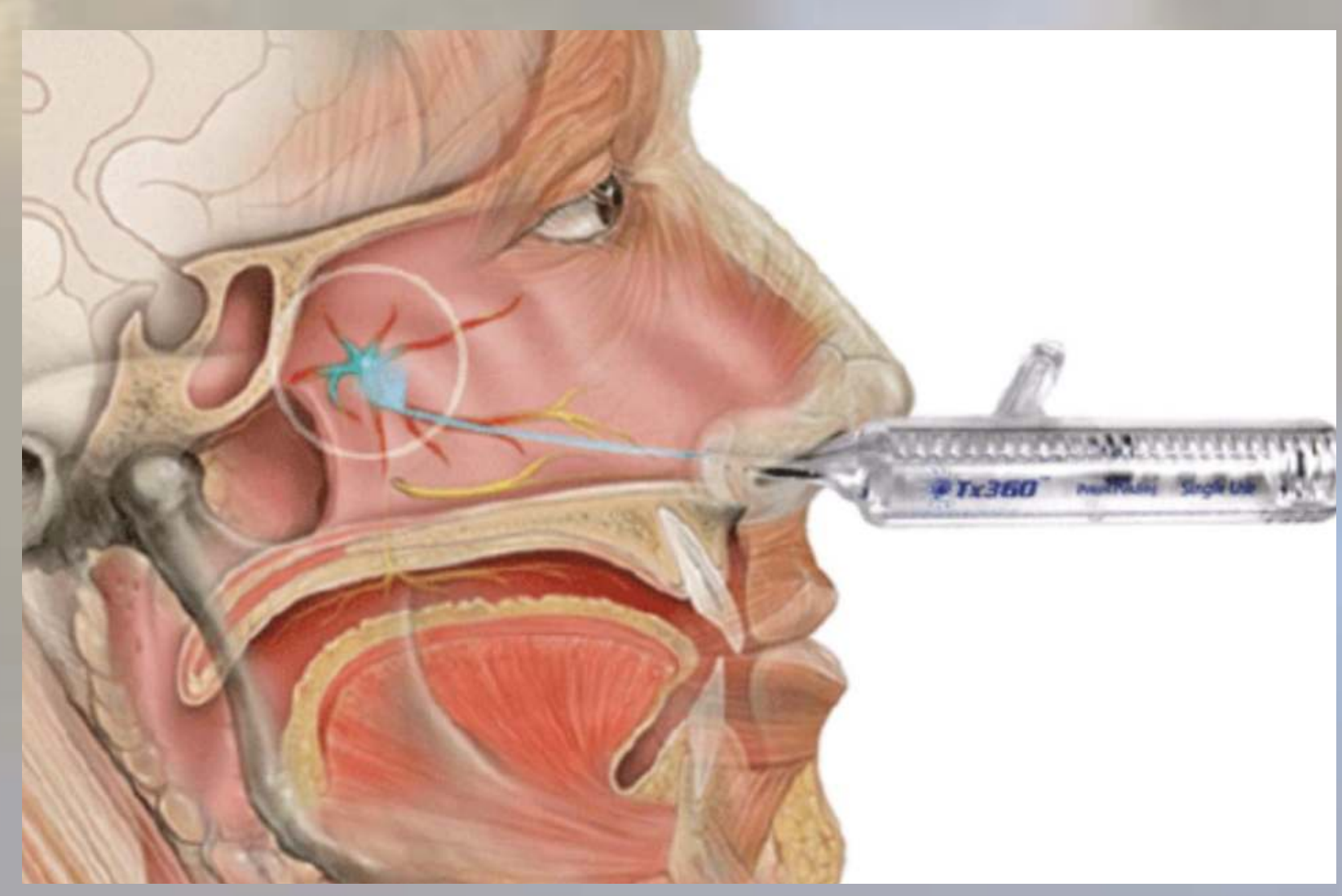

